\title{
Sprach- und kommunikationswissenschaftliche Analysen zu Energiekonzepten in Europa um 2014
}

\author{
Linguistic and communicational analysis \\ on energy concepts in Europe 2014
}

\begin{abstract}
The article is concentrated on the topics of linguistic and communicational analysis of near future energies among concepts of renewable energy sources in Europe 2014 based on visions according to Project Europe 2030. The analysis will be realized on the basis of text corpus of ZEIT.ONLINE and proved by the socio-empiric method SYMLOG as linguistic and communication research tool for systemic multilevel observation of groups dealing with the discourse of new energies. The article will show the various ways and debates within new linguistic and communicational analysis of the energy sources in the $\mathrm{EU}$ in general and as comparison between single $\mathrm{EU}$ states including Germany and Poland.

KEYWORDS: linguistic and communicational analysis, energy concepts, sources of renewable energy, Project Europe 2030, Symlog/method on systemic multilevel observation of groups, discourse of energies, EU communication.
\end{abstract}

\section{EINFÜHRUNG. ENERGIE-DISKURSE IN EUROPA 2014}

Die Frage der Energien bzw. Energieressourcen und der angrenzenden Aspekte dieser Fragestellung wird auf allen gesellschaftlichen Ebenen, in allen fachwissenschaftlichen und politischen Zentren, zahllosen Kommunikationsanalysen, Medien und in der breiten Öffentlichkeit sehr intensiv dis- 
kutiert. Das lässt sich anhand der Informationsanalysen (vgl. GoogleSchlagwort: energy mit ca. 1530000000 Ergebnissen [0,33 Sekunden - Stand am 10.02.15] und im Vergleich dazu das Google-Schlagwort: communication mit ca. 1080000000 Ergebnisse [0,28 Sekunden - Stand am 10.02.15]) vortrefflich zeigen, zumal von einer adäquaten Lösung der Energiefrage die Zukunft von allen Menschen abhängig ist. Man bezeichnet dieses Problem aus diesem Grund auch als ein globales Problem der Menschheit und stellt es als eine zivilisatorische Aufgabe in eine Reihe mit der weltweiten Eliminierung von Krankheit, Hunger und Krieg. Die Europäische Union hat sich in dieser Frage mit dem visionären Project Europe 2030 positioniert, das in diesem Artikel eine gesellschaftspolitische und interkulturelle Basis der EUKommunikation in den Energiefragen bildet:

Die EU muss eine gemeinsame Energiepolitik verfolgen, die sowohl nach innen als auch nach außen gerichtet ist und uns in die Lage versetzt, mehr Energieeffizienz und Energieeinsparungen zu erzielen, wie dies in der Strategie „Europa 2020“ gefordert wird, und unsere Energieversorgung aus Drittländern zu diversifizieren. Ferner müssen die Europäer ernsthaft diskutieren, inwieweit in Europa Bedarf an sicherer Kernenergie besteht, und sie müssen in Umrissen ein ständiges System von Anreizen für die Entwicklung alternativer Energiequellen festlegen.

(http://www.consilium.europa.eu/uedocs/cmsUpload/de_web.pdf, 2010: 6)

Wir befinden uns laut dieser Studie an der Schwelle einer neuen industriellen Revolution in Europa mit einer gemeinsamen Energiepolitik, geprägt von den Schlagworten, wie Effizienz, Sparsamkeit, Nachhaltigkeit und Diversifikation der Energieressourcen, die man aus heutiger Sicht wie folgt näher kennzeichnen kann:

Wenn nichts geschieht, wird die Energiesituation in Europa bis 2030 durch einen wachsenden Bedarf und ... eine rückläufige Versorgung gekennzeichnet sein. Die Abhängigkeit von kostspieligen Öl-, Gas- und ... Kohleeinfuhren aus Drittländern wird von derzeit $50 \%$ auf etwa $60 \%$ ansteigen, fossile Brennstoffe könnten bis zu $80 \%$ des europäischen Energiemix ausmachen. Obendrein werden die Lieferungen aus Gebieten stammen, die zu den politisch instabilsten der Welt zählen. Allein aufgrund ihrer Energieintensität sind unsere Volkswirtschaften äußerst gefährdet und anfällig für Turbulenzen. Damit nicht genug: Die Auswirkungen des Klimawandels auf die Weltwirtschaft könnten in ihrem Ausmaß die derzeitige Finanz- und Wirtschaftskrise noch übertreffen.

(http://www.consilium.europa.eu/uedocs/cmsUpload/de_web.pdf, 2010: 31)

Der Kommunikationsdiskurs der Energieressourcen in Europa zeichnet sich somit in der Gegenwart durch steigenden Verbrauch und sinkende Versorgung aus, womit zugleich auch die Lösungsmöglichkeiten aufgezeigt 
werden, die entweder auf die Modernisierung des Energieverbrauchs oder aber auf die Modernisierung der Versorgung oder aber auf alternative Energieressourcen außerhalb der bereits genutzten Energien hinaus laufen. Das zieht zugleich auch die problemgeladene Auseinandersetzung in der Frage nach der Verwirklichung von alternativen Energie-Konzepten nach sich und lässt den Grundtyp des Verhaltens der Kommunikationsteilnehmer am Energie-Diskurs in der Auseinandersetzung oder im Konsens deutlich werden, wobei wir uns vom folgenden zivilisatorischen Hexagon nach Senghaas (1995: 197) in der Lösung strittiger Fragen innerhalb der EU-Kommunikation leiten lassen:

Rechtsstaatlichkeit

Demokratische Prinzipien

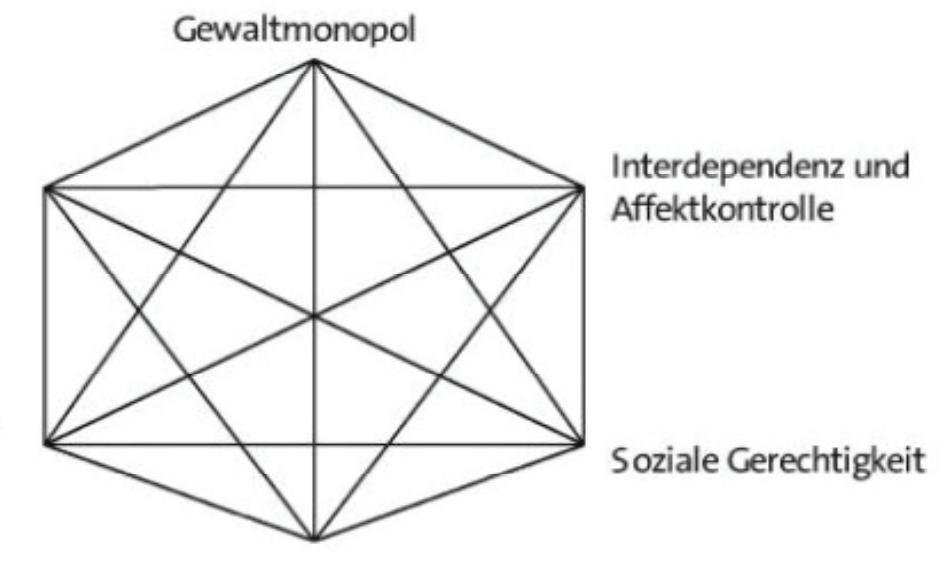

Konfliktkultur

Abb. 1. Zivilisatorisches Hexagon nach Senghaas <http:/ / www.konfliktbearbeitung.net/wiki/index.php5?title=Bild:Zivilisatorisches_Hex agon.jpg-> [Stand: 12.02.2015]

Die sprach- und kommunikationswissenschaftliche Untersuchung des Energie-Diskurses zeichnet sich durch Musterhaftigkeit der Texttypologien sowie durch die Autarkie und Autonomie der zugrunde liegenden Thematiken und Referenzsysteme aus. Deshalb ist ein systematischer Zugriff auf einen Textkorpus des Energie-Diskurses in einem Kommunikationsmedium notwendig, wobei mehrere methodenbasierte Analysemöglichkeiten zur Verfügung stehen. 


\section{TEXTKORPUS DES ENERGIE-DISKURSES IN DER ZEIT.ONLINE 2014}

Der Textkorpus des Energiediskurses der Gegenwart wird von verschiedenen Themenprofilen beherrscht, die man in unterschiedlichen Energieformen kommunikationswissenschaftlich zusammenfassen kann.

\section{1. Ökoenergie-Diskurse im Textkorpus}

Die Ökoenergie-Diskurse von 2014 zeichnen sich durch das Bemühen aus, die wichtigsten Energieressourcen der Gegenwart, wie etwa die atomaren und fossilen Energiequellen, durch neue bzw. erneuerbare Energiequellen zu ersetzen und dadurch eine grundlegende Änderung der Energiepolitik einzuleiten. Diese Energiediskurse sind also mit den Schlagworten Ökoenergien, erneuerbare Energien, Energiewende, Energierevolution schlechthin zu bezeichnen und korrespondieren mit den weltweiten Entwicklungstrends in den Sonnen-, Wind-, Wasser- und Wellenenergiequellen:

Das ist ein plausibles Argument. Dass Sonne und Wind in Europa inzwischen als ernst zu nehmende Energiequellen gelten, hat die Fantasie von Umweltschützern in Indien und China ungeheuer beflügelt. Andererseits berufen sich inzwischen auch australische Ökoreaktionäre auf das Beispiel der deutschen Energiewende: teuer und bringt nichts. Aber ist es nicht eine Flucht ins Grundsätzliche, Deutschlands konkretes Klimaziel für das Jahr 2020, wenn es außer Reichweite gerät, plötzlich nicht mehr so wichtig zu finden?

(http://www.zeit.de/2014/14/rainer-baake-atomausstieg/seite-2)

Die Ökoenergieformen werden nach Meinung von Experten eine notwendige Übergangsform in der weltweiten Umstellung der Energiewirtschaft einleiten, wenn alle Staaten nach einem Energie-Gipfel der UNO dieser Umstellung zustimmen. Gegenwärtig gibt es lediglich in einigen größeren Staaten, so auch in den EU-Staaten, eine rechtliche und technische Basis für eine solche technische Revolution in der Energiewirtschaft:

Wind- und Sonnenstrom, ergänzt durch Strom aus Gaskraftwerken, sollte die Kohle verdrängen und nebenbei die Atomkraft ersetzen - so war es geplant. Offenbar funktioniert dieser Plan nicht. Wenn der Ökostrom trotz seiner wetterabhängigen Schwankungen zuletzt überhaupt noch Fossilstrom ersetzt hat, dann hat er nicht den schmutzigsten, sondern den teuersten Energieträger aus dem Markt gedrängt: nicht Kohle, sondern Gas. Gehe das so weiter, warnen die Umweltschützer von Germanwatch, dann sei die "gesamte Konstruktion der Energiewende" in Gefahr. Was nützt es, immer mehr Grünstrom zu produzieren, wenn er den Kohlestrom nicht verdrängt?

(http:/ / www.zeit.de/2014/06/energiewende-sigmar-gabriel) 


\subsection{Atomenergie-Diskurse im Textkorpus}

Die Atomenergie-Diskurse sind in der jüngsten Zeit nach der Katastrophe im Atomkraftwerk in Fukushima/Japan (vgl. http://www.zeit.de/ wissen/umwelt/2011-03/fukushima-AKW-reaktoren) durch das Schlagwort "Atomausstieg" noch populärer geworden und werden durch die Atomenergiegegner in vielfältigen Protestaktionen in das Bewusstsein der Öffentlichkeit sowie der Politik getragen. Die Argumente der Atomenergielobby dagegen werden in den einzelnen EU-Ländern, wie z.B. Frankreich, Tschechien, Bulgarien und England, mit den Konzepten verknüpft, die auf die fehlende Effizienz und notwendige Netzwerke der erneuerbaren Energieressourcen verweisen und zugleich auch die heute überproportionalen Anteile der atomaren Energieressourcen am Gesamtenergiebudget der einzelnen EU-Staaten unterstreichen, wenn auch die Investitionen in die Atomkraftwerke enorm zu steigern sind:

\footnotetext{
Noch immer ist Atomkraft vor allem eine Energie der etablierten Industrienationen: 80 Prozent der weltweit installierten Leistung steht in OECD-Staaten. Mehr als drei Viertel gingen vor einem Vierteljahrhundert ans Netz. In der Regel sind sie auf eine Laufzeit von 40 Jahren ausgelegt.

Aufgrund ihres hohen Alters müssen nach Angaben der IEA bis zum Jahr 2040 weltweit 200 Atomkraftwerke abgeschaltet werden - knapp die Hälfte aller laufenden AKW. „Es wird eine große Herausforderung sein, diese Leistung zu ersetzen und eine bislang unvorhersehbare Zahl von Rückbauprojekten zu beginnen", sagt IEA-Chefvolkswirt Fatih Birol ZEIT ONLINE.

(http:/ / www.zeit.de/wirtschaft/2014-11/atomkraft-atomkraftwerk-iea)
}

\subsection{Energiemix-Diskurse im Textkorpus}

Die am häufigsten diskutierten Energieressourcen der Gegenwart stellen die unterschiedlichsten Mischungen bzw. Mix an Energien dar, die das jeweilige EU-Land gegenwärtig zur Verfügung hat, und in verschiedenen Energienetzen an die privaten und industriellen Verbraucher geliefert werden. Die z.Z. bekanntesten EU-Energiemixformen sind in folgenden Schlagworten erfassbar: Wasserenergie + Atomenergie + Gasenergie, Wasserenergie + Atomenergie + Kohleenergie oder aber Atomenergie + Solarenergie + Windenergie oder aber Atomenergie + Gezeitenenergie + Gasenergie oder aber ingenieurtechnische Mixformen mit Kopplung von Verbrauch und zugleich Produktion der Energien über z.B. Kraft-Wärme-Kraft-Konzepte (vgl. combined heat and power in Großbritannien oder kogeneracja energetyczna 
in Polen). Das birgt eine Vielzahl von unterschiedlichen Problemen bzw. Energieszenarien in sich und muss daher im nationalen und auch internationalen Rahmen ständigen Anpassungen in juristischer, technischer und kommerzieller Hinsicht unterzogen werden. Die Energiemixformen können daher in jedem einzelnen EU-Land lediglich geschätzt werden, was auch in den UNO-Ökogipfeln 2013 in Warszawa/Polen sowie in Lima/Peru 2014 deutlich zutage getreten ist, als es zu keiner weltweiten Einigung bei der Lösung der Klimaprobleme und folglich auch zu keiner Übergangslösung bei Energiemix auf dem Wege der weiteren $\mathrm{CO}_{2}$-Entlastung des Weltklimas gekommen ist:

In ihrem Report „POWE[R] 2030“ beschäftigen sich die Umweltschützer - basierend auf Modellrechnungen des Ingenieurbüros Energynautics - mit vier entscheidenden Fragen: Für wie viel Ökostrom wird das Netz der Zukunft ausgelegt sein? Wie muss der Umbau technisch aussehen? Wie viele zusätzliche Kapazitäten werden benötigt? Und vor allem: Was kostet das? Die Autoren der Studie vergleichen drei Szenarien. In ihrem Idealfall zettelt Europa eine „Energierevolution“ an, an der sich die 28 EUMitgliedsstaaten sowie Norwegen und die Schweiz beteiligen, und erreicht bis zum Jahr 2030 eine Ökostromquote von 77 Prozent. Im Referenzszenario läuft einfach alles weiter wie gehabt. Und im „Konfliktfall“ hängen Polen, Frankreich und Tschechien auch künftig an Nuklearenergie und Kohlekraft, während alle anderen Länder Wind- und Solarenergie ausbauen. Der ambitionierte Ausbau klappt nur unter bestimmten Bedingungen.

(http:/ / www.zeit.de/wirtschaft/2014-03/eu-gipfel-klimaziele-stromnetz-green peace)

Die Energiemixformen sind in technischer Hinsicht übergangsweise nach Meinung der Experten eine absolute Notwendigkeit, wenn man bedenkt, dass die neuen Energieformen auch neue Energienetze voraussetzen, was gegenwärtig nicht gegeben, sondern erst im Entstehen begriffen ist:

Allerdings, auch das geht aus der Studie hervor, ist die Revolution an Bedingungen
geknüpft. Ohne das richtige Netz und passende Prioritäten geht es nicht. Die
Energynautic-Ingenieure nehmen zum Beispiel an, dass Gas in Zukunft Vorrang vor
Kohle und Atomkraft hat, und dass Batterien etwa zehn Prozent der Solarstrom-
Kapazitäten speichern werden. Statt das gegenwärtige Drehstrom-Hochspannun-
gsnetz zu erweitern empfehlen sie, ein komplett neues Hochspannungs-Gleichstrom-
Netz darüber zu legen. Und sie weisen darauf hin, wie wichtig es ist, dass der zwi-
schen-staatliche Stromhandel technisch reibungslos funktioniert.
(http:// www.zeit.de/wirtschaft/2014-03/eu-gipfel-klimaziele-stromnetz-greenpeace)

Einige Energiemixformen prägen gegenwärtig die Energieressourcen einiger EU-Staaten in besonderer Weise, da sich diese Formen als effizient erweisen, und die Umstellung auf andere Energieformen bzw. eine Ener- 
giewende äußerst schwierig oder nur längerfristig und mit erheblichen Investitionen zu vollziehen ist. Dies trifft laut Kommunikationsanalysen der ZEIT.ONLINE insbesondere für solche großen EU-Länder wie Großbritannien, Frankreich und Polen zu:

\footnotetext{
Europa, das ist ein energiepolitischer Flickenteppich: Die Osteuropäer setzen vor allem auf die fossilen Energieträger, weil Kohle derzeit so günstig scheint. Die Skandinavier haben schon jetzt einen hohen Ökostromanteil - vor allem wegen der Wasserkraft. Großbritannien will Atomkraftwerke bauen, die Franzosen planen dagegen, langfristig aus der Kernenergie auszusteigen.

Der Nachbar im Osten sperrt sich schon seit Jahren gegen zu hohe Auflagen beim Klimaschutz. Der Grund ist simpel: Das Land gewinnt rund 90 Prozent seines Stroms aus Kohle. Gleich zu Beginn ihrer Amtszeit zeigten die Kohlekumpel in Polen der neuen Ministerpräsidentin Ewa Kopacz, was sie von ihr erwarteten: Sie demonstrierten für den Erhalt ihrer Arbeitsplätze. Die vier großen, staatlichen Bergbauunternehmen in Polen stehen unter immensem Druck, weil sie immer weniger Gewinn machen.

(http://www.zeit.de/wirtschaft/2014-10/eu-gipfel-energie-klima)
}

Als sprach- und kommunikationswissenschaftliches Zwischenfazit lassen sich bei den Energiemix-formen die Schlagworte Energieeffizienz, Energiewende, Energierevolution und Energietransition (vgl. transition énergétique in Frankreich oder transformacja energetyczna in Polen) fest machen.

\section{SYMLOG-METHODE IN DER UNTERSUCHUNG DER ENERGIEDISKURSE DER ZEIT.ONLINE 2014}

Die Symlog-Methode als ein begriffliches Akronym von "Systematic Multiple Level Observation of Groups" stellt einen Raster zur Beobachtung von Sprachverhalten und Handlungen der Gruppenteilnehmer dar und zielt auf die Probleme ab, die eine Gruppe bewältigen muss, um eine Aufgabe, z.B. Kommunikationsdiskurse in der Energiefrage, erfolgreich zu erfüllen (vgl. Bales/Cohen 1982: 28). Aus diesem Grunde werden die Gruppenprobleme jeweils zwei Bereichen zugeordnet: (a) dem sozial-emotionalen Bereich mit drei Kategorien, die der emotionalen Integration der Mitglieder dienen, um Spannungen beim Zusammenwirken zu bewältigen und Entscheidungen zu treffen sowie (b) dem Aufgabenbereich mit drei Kategorien, die zur Analyse der Orientierung, Bewertung und Kontrolle von Sprachverhalten und Handlungen der Gruppenmitglieder dienen. 
Auf der anderen Seite wird die Symlog-Methode als eine qualitative Textanalysemethode in der Textologie, Narratologie, Sozialwissenschaft und in der Angewandten Linguistik für die Zwecke der Textinterpretation in semantisch-pragmatischer Hinsicht genutzt, wobei es auch zu Kombinationen von Symlog und mathematisch-statistischen Verfahren kommt, was die Repräsentativität, Validität, Signikanz und Reliabilität der dabei gewonnenen sprachlichen und kommunikativen Ergebnisse wesentlich steigert. Man spricht deshalb in diesem Zusammenhang von einer auf den Textkorpus bezogenen, angewandt-linguistischen Betrachtung von Sprach- und Kommunikationsdaten.

In diesem Sinne lässt sich die Symlog-Methode in die Agenda-SettingHypothese der Kommunikation einordnen, welche besagt, dass man die Merkmale des sprachlichen und kommunikativen Verhaltens in Form vom Texten untersuchen kann, die in den Medien bzw. Medientexten über eine gewisse Frequenz (anhand der Schlagworte bzw. Kontextsynonyme) und Verteilung (Einschaltquotensysteme oder Nachrichtenselektionsprogramme in den Medien (vgl. Möller 2005) verfügen und damit nachweislich viele Menschen bewegen, weil „die von den Medien besonders hervorgehobenen Themen auch vom Publikum als die aktuell wichtigen Themen aufgefasst werden“ (Früh 1991: 177, vgl. auch Maletzke 1981: 18ff.), was am folgenden Symlog-Modell deutlich wird:

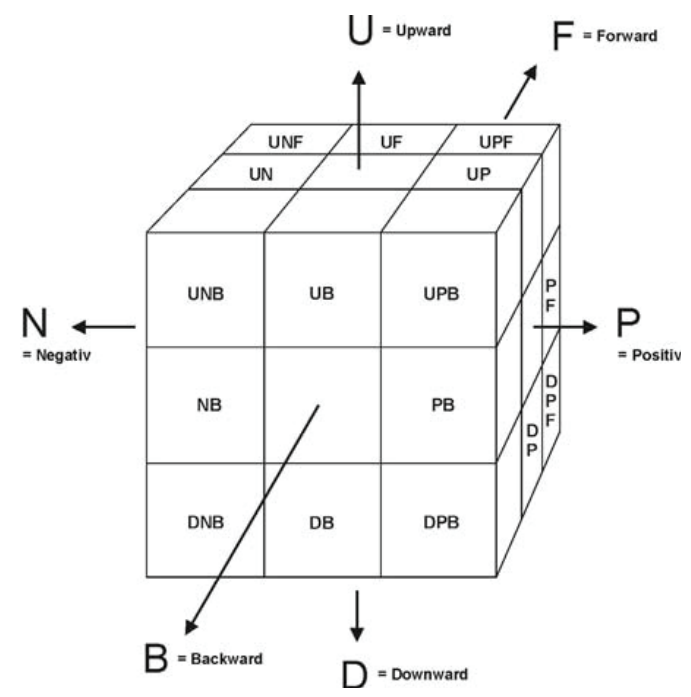

Abb. 2. Symlog-Dimensionen-Modell von Robert F. Bales und Stephen Cohen (http://trac.edfac.usyd.edu.au/uchemnitz_new/attachment/wiki/wiki_gruppe2_aufga be2/-symlog_dimensionen.jpg, vgl. auch Titscher et all. 1998: 175ff.) 
Damit postuliert die Symlog-Methode, dass die Interaktionen zu Themen bzw. Textinhalten in einem dreidimensionalen Wahrnehmungs- und Bewertungsraum mit jeweils zwei Ausprägungen und mit insgesamt 26 Raumpositionen (Position $27=$ Beobachter) analysiert werden können, die man wie folgt interpretiert:

- Einflussnahme vs. Verzicht auf Einflussnahme (Upward - Downward),

- Emotionale Zuwendung vs. Emotionale Distanzierung (Postive - Negative),

- Kooperation-Zielgerichtetheit vs. Impulsivität-Gefühlsbestimmtheit (Forward - Backward).

Auf diese Weise wird deutlich, dass die Kommunikationsdiskurse zu Energiefragen auf der Basis des Textkorpus der ZEIT.ONLINE mit der Symlog-Methode zumindest in den Wirkungen der einzelnen Gruppenhandlungen modelliert werden können. Dies ist für die Kommunikationsdiskurse bzw. Textanalysen zu Energiefragen besonders zutreffend, weil es in der Energiekommunikation keine Einzelgänge bzw. Einzelhandlungen von beteiligten Personen, sondern vielmehr und über größere Zeiträume hinweg, so auch 2014, konkrete Gruppendiskurse sowie auch medienöffentliche, fachwissenschaftliche und gesellschaftspolitische Konzepte gibt.

Die kollektive Behandlung der Energiediskurse ist demzufolge eine Realität auf der Basis der Fakten und bedingt deshalb eine erfolgreiche Lösung der Energiefrage in Europa und auch anderswo in direkter Weise und das nachhaltig. Das Symlog-Modell rastert die Gruppeinteraktionen der beteiligten Kammunikanten in Wirkungsprofile, Bündelungen von möglichen Wirkungen, die man innerhalb eines semantisch-pragmatischen Verfahrens (vgl. Typologie von Merten 1999: 17ff) vermittels des Semantischen Atlass der Symlog-Profile (vgl. Orlik, Schario 1989: 21ff.) auch interpretieren kann.

Die Interpretation der Wirkungsprofile der Energiediskurse anhand des Textkorpus der ZEIT.ONLINE wird uns im nächsten Punkt ausführlich beschäftigen.

\section{DIE WIRKUNGSPROFILE DER ENERGIEDISKURSE IM TEXTKORPUS DER ZEIT.ONLINE 2014}

Um die Wirkungsprofile der Energiediskurse einer SymlogUntersuchung zuzuführen, bedarf es zunächst der Ermittlung von typischen Schlagworten in der Lösung strittiger Fragen. Hierbei bedienen wir uns der konsensgeladenen Schlagworte im untersuchten Textkorpus der Energiedis- 
kurse der ZEIT.ONLINE 2014, weil die Energiediskurse eine Vielzahl von gemeinsamen, kollektiven Lösungen enthalten und deshalb auch im Einvernehmen/Konsens aller beteiligten Seiten gestaltet werden.

\subsection{Konsensgeladene Schlagworte im Textkorpus der Energiediskurse}

Die konsensgeladenen Schlagworte im Textkorpus der Energiediskurse werden zunächst auf der Basis von www.duden.de und www.synonyme.de ermittelt und mit den vorhandenen Textsynonymen aus dem Textkorpus der ZEIT.ONLINE verglichen, um deren Frequenz und Verteilung in den Texten näher $\mathrm{zu}$ erfassen. Im Einzelnen sind es laut http://synonyme. woxikon.de/synonyme/konsens.php folgende Schlagworte:

Bedeutung: Einhelligkeit [n] Einigkeit, Einmütigkeit, Einvernehmen, Übereinkommen, Anerkennung, Eintracht, Einverständnis, Erlaubnis, Gemeinsamkeit, Gleichsinn, Plazet, Konsens

Bedeutung: Einigkeit [n] Einklang, Eintracht, Einvernehmen, Harmonie, Übereinstimmung, Konsens, Einhelligkeit

Bedeutung: Erlaubnis [n] Bestätigung, Einverständnis, Einwilligung, Genehmigung, Bejahung, Bekräftigung, Freibrief, Konsens, Sanktion, Plazet

Bedeutung: Übereinstimmung [n] Einigkeit, Einvernehmen, Konsens, Harmonie, Einverstandensein, Einverständnis

Bedeutung: Abkommen [n] Übereinkunft, Abschluss, Ausgleich, Einigung, Handel, Konsens, Pakt, Vereinbarung, Übereinkommen

Bedeutung: Verbundenheit [n] Gemeinsamkeit, Geistesverwandtschaft, Gemeinschaft, Miteinander, Partnerschaft, Solidarität, Ähnlichkeit, Verwandtschaft, Zusammengehörigkeit, Einhelligkeit, Übereinstimmung, Konsens, Gemeinschaftlichkeit, Verbindung, Zusammenarbeit, Berührungspunkt.

Unter Hinzunahme von Synonyma der Duden-Redaktion (http://www. duden.de/rechtschreibung/Konsens) in den Bedeutungen (a) Übereinstimmung der Meinungen und (b) Zustimmung/Einwilligung entsteht die folgende Ergänzung der obigen Liste:

Billigung, Einigkeit, Einklang, Einmütigkeit, Eintracht, Einvernehmen, Einverständnis, Einwilligung, Erlaubnis, Gemeinschaft, Genehmigung, Harmonie, Okay, Plazet, Sanktion, Solidarität, Übereinstimmung, Zusage, Zusammenhalt, Zustimmung.

Schaut man sich diese Listen näher an, so stellt man eine gewisse Überscheidung der semantischen und pragmatischen Spektren von Konsens im Energiediskurs fest, was zunächst auf die unterschiedliche Interpretation der wesentlichen gegenständlichen Diskursbestandteile des Konsenskonzeptes 
hinweist (vgl. Tschentscher 2002: 43ff.) Dieser Widerspruch kann entweder durch ein sachlogisches Verfahren oder aber durch Kontext gelöst werden, weil dem Kontext auch eine monosemierende wie auch eine systematisierende Funktion zukommt, was den begrifflichen Kern eines Schlagwortes durch Textstellen eindeutig macht, zumal diese Kontext-Schlagworte in der modernen Textologie eines Hypertextes durch Link gestützt sind, wie es am folgenden Text der Energiediskurse der ZEIT.ONLINE mit dem Titel „Die stille Kraft, seit dreißig Jahren" (http:// www.zeit.de/2014/14/rainer-baakeatomausstieg/seite-2) deutlich wird:

a) „Drohen nicht ständig Blackout und Krise als Folgen einer angeblich überstürzten Energiewende?"

b) „Baake hat zuletzt als Leiter des Thinktanks Agora Energiewende Konzepte mit entwickelt, die weit über das grüne Spektrum hinaus Aufmerksamkeit und oft auch Zustimmung gefunden haben."

c) "Als in Fukushima die Reaktoren absoffen und explodierten, rief er im Umweltministerium an, um an eine alte Sicherheitsanalyse zu erinnern sie betraf genau jene deutschen Atomkraftwerke, die dann tatsächlich zuerst abgeschaltet wurden."

Auf Grundlage der vorangegangenen Schlagwortanalyse lassen sich unter Hinzunahme der Kontexte folgende prototypische Schlagworte mit besonders großen Wirkungsprofilen in Bezug auf Häufigkeit und Verteilung im Textkorpus der konsensgeladenen Energiediskurse der ZEIT.ONLINE 2014 im Diagramm darstellen:

\section{Energiediskurse}

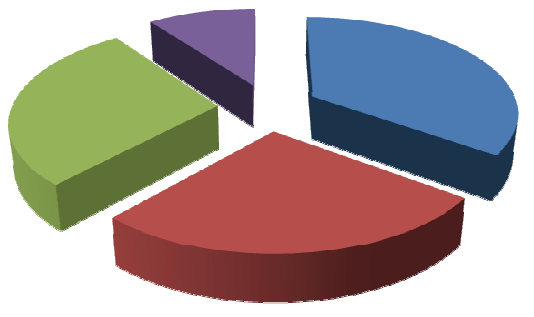

口übereinkommen

Abkommen

Kooperation

- Gemeinschaft

Abb. 3. Eigene Darstellung der prototypischen Schlagworte in den konsensgeladenen Energiediskursen im Textkorpus der ZEIT.ONLINE 2014

Die Listen der konsenstragenden prototypischen Schlagworte werden in den wertenden Bestandteilen um prototypische Adjektive mit sozial- 
emotionalen Wirkungen in Anlehnung an Duden-Diskurse (http://www. duden.de/rechtschreibung/Konsens) erweitert, um auf diese Weise gemäß Symlog-Methode eine Positionierung der konsensgeladenen Schlagworte in den Energiediskursen der ZIET.ONLINE 2014 wie folgt zu erhärten: breit, gesellschaftlich, überparteilich.

\section{ZUSAMMENFASSUNG}

Die vorangestellten sprach- und kommunikationswissenschaftlichen Analysen haben eine Vielzahl von konsensgeladenen Wirkungsprofilen in den Energiediskursen der ZEIT.ONLINE 2014 aufgezeigt, die eine intensive und zugleich auch schöpferische Auseinandersetzung mit den Entwicklungen in den Energieressourcen der EU-Länder mit weitverzweigten semantisch-pragmatischen Konzepten dokumentieren.

Die sprach- und kommunikationswissenschaftlichen Analysen haben die These über eine gemeinsame Agenda der Energiediskurse der EU-Länder insoweit gestützt und die gemeinsamen prototypischen Schlagworte/Kontextsynonyme wie auch die gemeinsamen prototypischen Bewertungen/Adjektive in den EU-Energiediskursen sowohl in den Energiekonzepten als auch in deren sprachlichen Ausprägungen nachgewiesen.

\section{LITERATURVERZEICHNIS}

Bales, R.F., Cohen, S.P. 1982. SYMLOG. Ein System für die mehrstufige Beobachtung von Gruppen. Stuttgart: Klett-Cotta.

Bericht der Reflexionsgruppe an den Europäischen Rat über die Zukunft der EU 2030, 2010. Brüssel.

Früh, W., 1991. Medienwirkungen. Das dynamisch-transaktionale Modell. Theorie und empirische Forschung. Opladen: Westdeutscher Verlag.

<http://synonyme.woxikon.de/synonyme/konsens.php>, [abgerufen am: 19.02.2015].

<http://trac.edfac.usyd.edu.au/uchemnitz_new/attachment/wiki/wiki_gruppe2_aufga be2/symlog_dimensionen.jpg >, [abgerufen am: 19.02.2015].

<http://www.consilium.europa.eu/uedocs/cmsUpload/de_web.pdf>, [abgerufen am: 12.02.2015].

<http://www.duden.de/rechtschreibung/Konsens>, [abgerufen am: 19.02.2015].

<http://www.konfliktbearbeitung.net/wiki/index.php5?title=Bild:Zivilisatorisches_Hex agon.jpg->, [abgerufen am: 12.02.2015].

<http://www.zeit.de/2014/06/energiewende-sigmar-gabriel>, [abgerufen am:12.02. 2015].

<http://www.zeit.de/2014/14/rainer-baake-atomausstieg/seite-2>, [abgerufen am: 19.02.2015]. 
<http:/ / www.zeit.de/wirtschaft/2014-03/eu-gipfel-klimaziele-stromnetz-greenpeace>, [abgerufen am: 12.02.2015].

<http://www.zeit.de/wirtschaft/2014-10/eu-gipfel-energie-klima>, [abgerufen am: 12.02.2015].

<http://www.zeit.de/wirtschaft/2014-11/atomkraft-atomkraftwerk-iea>, [abgerufen am: 12.02.2015].

Maletzke, G., 1981. Medienwirkungsforschung. Grundlagen, Möglichkeiten, Grenzen. Tübingen: Niemeyer Verlag.

Merten, K., 1999. Einführung in die Kommunikationswissenschaft. Bd. 1. Grundlagen der Kommunikationswissenschaft. Münster: LIT-Verlag.

Möller, E., 2005. Die heimliche Medienrevolution. Wie Weblogs, Wikis und freie Software die Welt verändern. Hannover: Heise Zeitschriften Verlag GmbH \& Co. KG.

Orlik, P., Schario, R., 1989. Die Analyse sozialer Interaktionsfelder aus der Romanliteratur. London: Sage Publications Ltd.

Senghaas, D., 1995. Frieden als Zivilisierungsprozess. In: Senghaas D. (Hrsg.). Den Frieden denken. Si vis pacem, para pacem. Frankfurt/Main: Suhrkamp Verlag, 196-223.

Titscher, S., 1998. Methoden der Textanalyse, Leitfaden und Überblick. Opladen: Westdeutscher Verlag.

Tschentscher, A., 2002. Der Konsensbegriff in Vertrags- und Diskurstheorien (PDF; 701 kB). In: Rechtstheorie, 34, 43-59. 
